\title{
Crystal structure and phase diagrams of iron-based superconductors
}

\author{
Xigang Luo ${ }^{1,2}$ and Xianhui Chen ${ }^{1,2^{*}}$
}

\begin{abstract}
Since the discovery of high-temperature superconductivity (HTS) in iron-based compounds, a variety of systems with different spacer layers have been fabricated. Concurrently, considerable experimental and theoretical effort has been expended exploring the characteristics and source of HTS in iron-based superconductors. However, the origin of this HTS remains unresolved to date, while considerable debate exists regarding the underlying physics of the normal-state properties of iron-based compounds in particular. In this short review, we will briefly summarize the crystal structures and phase diagrams of the iron-based superconducting systems, aiming to discover potential avenues for the development of new superconductors with higher superconducting transition temperatures $\left(T_{c}\right)$, along with indications of the specifics of the HTS mechanism in these substances.
\end{abstract}

In January 2008, Kamihara et al. [1] reported F-doped LaOFeAs iron-oxypnictide, a new superconductor with a superconducting transition temperature of $T_{c} \sim 26 \mathrm{~K}$. Soon after, Chen et al. [2] reported a new superconductor, Sm$\mathrm{FeAsO}_{1-x} \mathrm{~F}_{x}$, and elevated its $T_{\mathrm{c}}$ to $43 \mathrm{~K}$ under ambient pressure. It manifests the strong possibility that even higher $T_{c}$ values may be obtained in such layered oxypnictides. The observed $T_{c}$ of $43 \mathrm{~K}$ in $\mathrm{SmFeAsO} \mathrm{A}_{1-x} \mathrm{~F}_{x}$ exceeds the theoretical upper limit $(39 \mathrm{~K}$ ) predicted by the Bardeen-Cooper-Schrieffer (BCS) theory, providing a strong argument for considering layered iron-based superconductors as unconventional superconductors. In this article, we will review the crystal structures, synthetic techniques, and phase diagrams of iron-based superconductors.

\section{CRYSTAL STRUCTURE}

As early as 2006, Kamihara et al. [3] reported superconductivity in LaOFeP, which has the same cyrstallographic structure as $\mathrm{LaFeAsO}$, but the value of $T_{\mathrm{c}}$ was only $4 \mathrm{~K}$ at that point. However, $T_{\mathrm{c}}$ can be significantly increased by F-doping, as had already been shown for the $\mathrm{LaFePO}_{0.94} \mathrm{~F}_{0.06}$ system, which has a $T_{c}$ of $7 \mathrm{~K}$ [3]. At that time, the $T_{\mathrm{c}}$ of these compounds was too low to attract considerable scien- tific interest, until the discovery of $\mathrm{LaFeAsO}_{1-x} \mathrm{~F}_{x}$ and Sm$\mathrm{FeAsO}_{1-x} \mathrm{~F}_{x}[1,2,4,5]$. Subsequently, a large amount of new iron-based superconductors were discovered, which can be classified into several families according to their structural characteristics, as shown in Fig. 1. The iron-based superconductors share a common anti-PbO-type $\mathrm{Fe}_{2} \mathrm{X}_{2}(\mathrm{X}=$ $\mathrm{P}, \mathrm{As}, \mathrm{Se}, \mathrm{Te}$, etc.) layer within their structures. This $\mathrm{Fe}_{2} \mathrm{X}_{2}$ layer consists of edge-shared $\mathrm{FeX}_{4}$ tetrahedra, and the discovered iron-based superconductors can be regarded as being comprised of an alternative stacking of the $\mathrm{Fe}_{2} \mathrm{X}_{2}$ layer along with spacer slabs. Considering the different structural characteristics of the spacer slabs, the iron-based superconductor can be classified using the following analogues:

(I) The 11 system stacking with only anti-PbO $\mathrm{FeCh}_{2}$ layers, where $\mathrm{Ch}=\mathrm{Se}, \mathrm{Te}, \mathrm{S}$, and their combinations. Bulk FeSe can behave as a superconductor below approximately $10 \mathrm{~K}$ under ambient pressure [6], and the value of $T_{\mathrm{c}}$ is increased to $37 \mathrm{~K}$ through the application of external pressure $[7,8]$. By mixing Se and $\mathrm{Te}, T_{\mathrm{c}}$ is increased to $15 \mathrm{~K}[9,10]$;

(II) The 111 system, which possesses an anti-PbFCltype structure. Examples include LiFeP, LiFeAs, and $\mathrm{Na}$ FeAs [11-13]. LiFeAs and $\mathrm{NaFeAs}$ are superconducting when free of doping [12,13]. In the case of NaFeAs, however, only filamentary superconductivity exists, and bulk superconductivity requires doping with $\mathrm{Co}$ or $\mathrm{Cu}$ [14-16];

(III) The 122 system, which has a $\mathrm{ThCr}_{2} \mathrm{Si}_{2}$-type structure. Examples include $\mathrm{AFe}_{2} \mathrm{As}_{2}(\mathrm{~A}=\mathrm{Ba}, \mathrm{Sr}, \mathrm{Ca}, \mathrm{Eu}, \mathrm{K}$, $\mathrm{Rb}$, and Cs) [17-20] and $\mathrm{AFe}_{2} \mathrm{Se}_{2}(\mathrm{~A}=\mathrm{Na}, \mathrm{K}, \mathrm{Rb}, \mathrm{Cs}, \mathrm{Tl}$, etc.) [21-24]. Superconducting 122 arsenides with other structure types also exist, such as $\mathrm{SrPt}_{2} \mathrm{As}_{2}$, which has a $\mathrm{CaBe}_{2} \mathrm{Ge}_{2}$-type structure [25], and $\mathrm{BaPd}_{2} \mathrm{As}_{2}$, with a CeMg $\mathrm{Si}_{2}$-type structure [26]. The highest $T_{\mathrm{c}}$ of 122 arsenides is $38 \mathrm{~K}$, which is realized in $\mathrm{K}$ - or Rb-doped $\mathrm{BaFe}_{2} \mathrm{As}_{2}$ or Sr$\mathrm{Fe}_{2} \mathrm{As}_{2}[17,20]$. The intercalated FeSe superconductors also crystallize in a $\mathrm{ThCr}_{2} \mathrm{Si}_{2}$-type structure, including $\mathrm{A}_{x} \mathrm{Fe}_{2-y}$ $\mathrm{Se}_{2}$ (with Fe vacancy in the FeSe layers) [21-24] and their derivative Fe-vacancy-free compounds, $\mathrm{A}_{x}\left(\mathrm{NH}_{3}\right)_{z} \mathrm{Fe}_{2} \mathrm{Se}_{2}$ or $\mathrm{A}_{x}\left(\mathrm{NH}_{2}\right)_{y}\left(\mathrm{NH}_{3}\right)_{z} \mathrm{Fe}_{2} \mathrm{Se}_{2}(\mathrm{~A}=\mathrm{Li}, \mathrm{Na}, \mathrm{K}, \mathrm{Ba}$, etc.) [27-29];

\footnotetext{
${ }^{1}$ Hefei National Laboratory for Physical Sciences at Microscale and Department of Physics, University of Science and Technology of China, Hefei 230026, China

${ }^{2}$ Key Laboratory of Strongly-coupled Quantum Matter Physics, Chinese Academy of Sciences, University of Science and Technology of China, Hefei 230026, China

"Corresponding author (email: chenxh@ustc.edu.cn)
} 


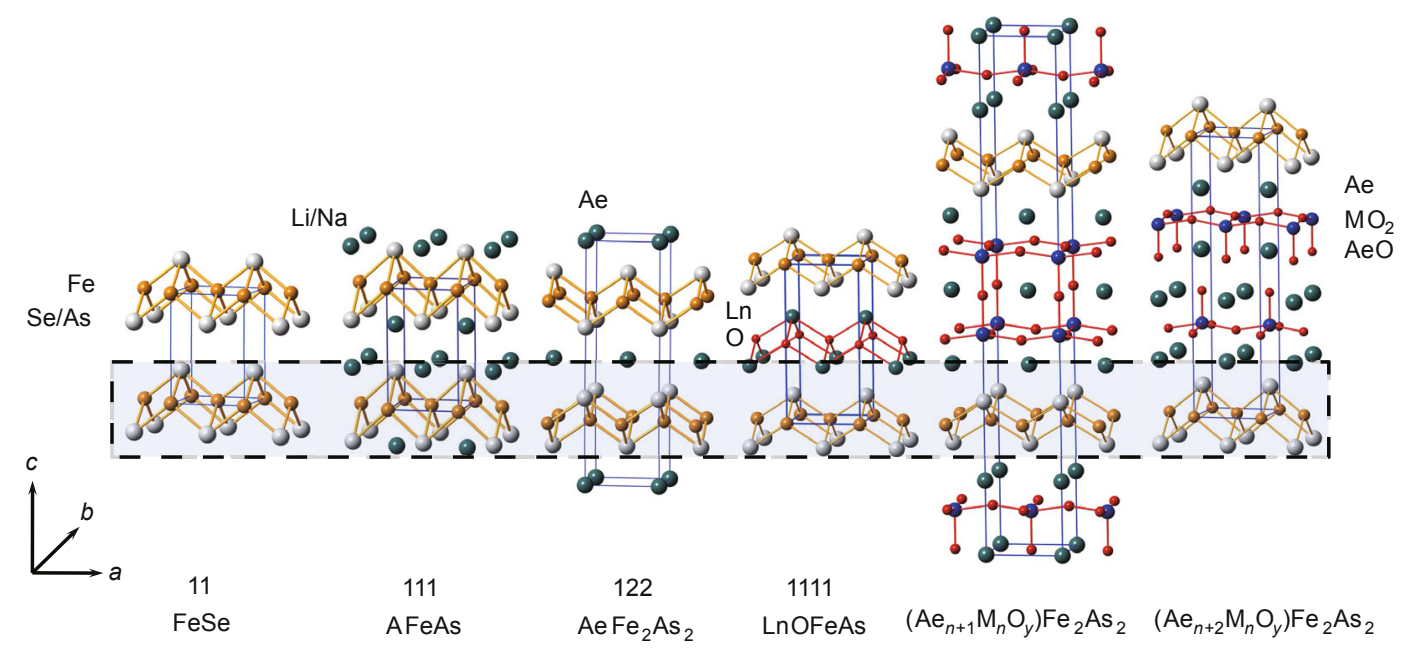

Figure 1 Schematic view of the crystal structures of several typical iron-based superconductor families, in which A, Ae, Ln, and M represent alkali, alkali earth, lanthanide, and transition metal atoms, respectively.

(IV) The 1111 system with ZrCuSiAs-type structure. Examples include $\mathrm{LnOFeAs}(\mathrm{Ln}=$ rare earth metal) $[1,2,4,5]$, AeFFeAs $(\mathrm{Ae}=\mathrm{Ca}, \mathrm{Sr}, \mathrm{Ba}$, and $\mathrm{Eu})[30,31]$, and CaHFeAs [32]. The highest bulk $T_{\mathrm{c}}(55 \mathrm{~K})$ was achieved for the F-doped SmOFeAs or Sm-doped SrFFeAs systems $[4,5,33]$;

(V) Systems with perovskite-like blocks intercalated between FeAs layers, such as $\mathrm{Ae}_{n+1} \mathrm{M}_{n} \mathrm{O}_{y} \mathrm{Fe}_{2} \mathrm{As}_{2}$ ( $n$ perovskite layers sandwiched between the adjacent FeAs layers and $y$ $3 n-1$ ) [34-36], and $\mathrm{Ae}_{n+2} \mathrm{M}_{n} \mathrm{O}_{y} \mathrm{Fe}_{2} \mathrm{As}_{2}[37,38]$ ( $n$ perovskite layers plus one roclk-salt layer in each blocking layer between the adjacent FeAs layers, $y \sim 3 n$ ). A $T_{c}$ of greater than $40 \mathrm{~K}$ has been observed in these compounds [36];

(VI) Systems with skutterudite intermediary layers, which have been identified as $\mathrm{Ca}_{10}\left(\mathrm{Pt}_{3} \mathrm{As}_{8}\right)\left(\mathrm{Fe}_{2} \mathrm{As}_{2}\right)_{5}$ (10-38) (referred to as $\alpha$-phase) and $\mathrm{Ca}_{10}\left(\mathrm{Pt}_{4} \mathrm{As}_{8}\right)\left(\mathrm{Fe}_{2} \mathrm{As}_{2}\right)_{5}(10-4-$ 8) (referred to as $\beta$-phase) $[39,40]$;

(VII) The FeSe-derived superconductor with completely new $\left(\mathrm{Li}_{0.8} \mathrm{Fe}_{0.2}\right) \mathrm{OH}$ spacer layer blocks between the adjacent FeSe layers, $\left(\mathrm{Li}_{0.8} \mathrm{Fe}_{0.2}\right) \mathrm{OHFeSe}$ [41-43]. This superconductor exhibits a $T_{c}$ of as high as $43 \mathrm{~K}$. In this structure, the FeSe layer is the conducting block, while the $\left(\mathrm{Li}_{0.8} \mathrm{Fe}_{0.2}\right) \mathrm{OH}$ layer is the charge reservoir block [42]. With alternate stacking of the $\left(\mathrm{Li}_{0.8} \mathrm{Fe}_{0.2}\right) \mathrm{OH}$ and anti-PbO-type FeSe layers, there exists a weak hydrogen bonding interaction between the layers (with quite a large $\mathrm{H}$-Se distance of $3.078 \AA$ ) [42];

(VIII) Other composite structures, such as the $\mathrm{BaTi}_{2} \mathrm{As}_{2} \mathrm{O}$ and $\mathrm{BaFe}_{2} \mathrm{As}_{2}$ composited $\mathrm{Ba}_{2} \mathrm{Ti}_{2} \mathrm{Fe}_{2} \mathrm{As}_{4} \mathrm{O}$ superconductor [44].

Detailed crystallographic information for these classes of compounds can be found in previous reviews (for instance, Ref. $[45,46])$. Besides the classification according to the chemical formulae discussed above, the majority of the iron-based superconductors can be divided into two larger classes based on the Bravais lattice, i.e., groups of tetragonal $(11,111,1111$, etc.) and body-centered tetragonal (122) structures. Among these substances, the electronic properties of body-centered tetragonal (122) materials tend to be more three-dimensional. Note that the compounds with perovskite-like blocks can have either tetragonal or body-centered tetragonal structures, depending on the presence of a rock-salt layer.

As a result of the large amount of available data concerning crystal structure and the corresponding $T_{c}$ for ironbased superconductors, a specific relationship between the structure parameters and $T_{c}$ was found $[47,48]$. The bond angle $(\alpha)$ of As-Fe-As, which reflects the distortion of the $\mathrm{FeAs}_{4}$ tetrahedron, was thought to be closely related to the superconductivity of this material [47]. As shown in Fig. 2a, the maximum $T_{c}$ in a FeAs-based superconducting system was achieved when the FeAs 4 tetrahedron was perfectly regular, with $\alpha=109.47^{\circ}$. However, $\mathrm{Fe}(\mathrm{Se}, \mathrm{Te})$ does not follow this rule, and the maximum $T_{\mathrm{c}}$ is obtained for $\alpha \sim 100.8^{\circ}$. Also, among the intercalated FeSe superconductors, $\mathrm{Li}_{0.6}\left(\mathrm{NH}_{2}\right)_{0.2}\left(\mathrm{NH}_{3}\right)_{0.8} \mathrm{Fe}_{2} \mathrm{Se}_{2}$ and $\left(\mathrm{Li}_{0.8} \mathrm{Fe}_{0.2}\right) \mathrm{OHFeSe}$, which exhibit values of $T_{c}$ higher than $40 \mathrm{~K}$, have values of $\alpha=102.93(6)^{\circ}(\times 2)$ and $103.2(2)^{\circ}(\times 2)$, respectively $[28,42]$, which are less than those observed for $\beta-\mathrm{Fe}_{1+\delta} \mathrm{Se}\left(103.9^{\circ} \times 2\right)$ [49]. These facts suggest that distortion of the tetrahedron in FeSe-derived superconductors could enhance the superconductivity [42], in contrast to the concept that the ideal $\mathrm{FeAs}_{4}$ tetrahedron is preferable for superconductivity in FeAs-based superconductors. Another typical relationship is the dependence of $T_{c}$ on the height of the anion (As, P, Se, and Te) from the Fe layer $(h)$, as shown in Fig. 2b [48]. The value of $h$ depends on the anion type, and increases in or- 

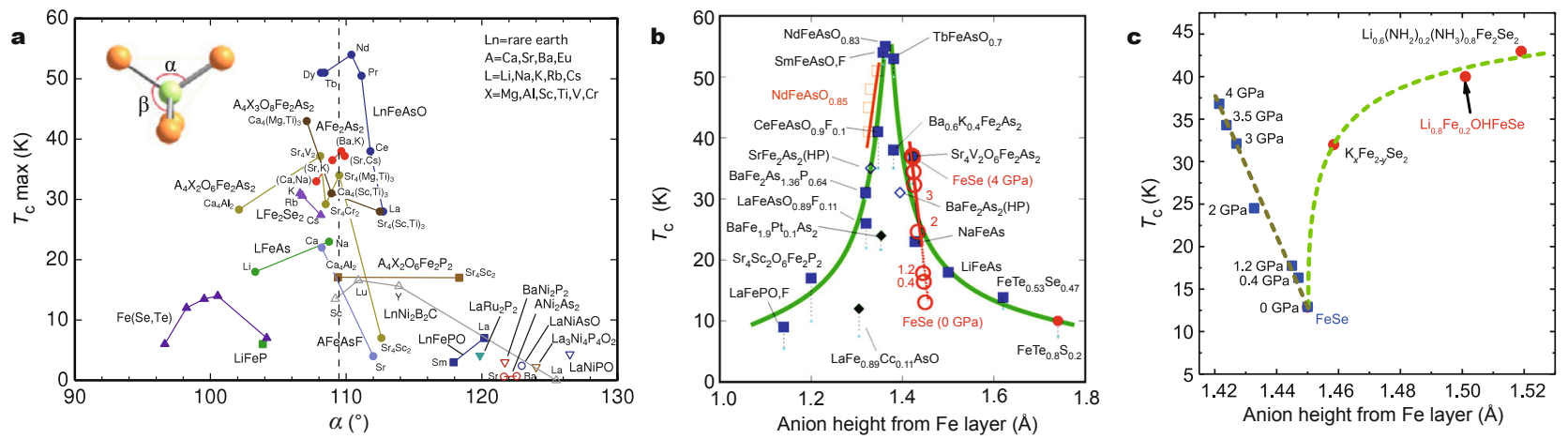

Figure 2 (a) Relationship between the As(top)-Fe-As(top) bond angle, $\alpha$, and the $T_{\mathrm{c}}$ of iron-based superconductors. Reprinted with permission from Ref. [47] (Copyright 2012, Elsevier). (b) General anion height dependence of $T_{\mathrm{c}}$ for iron-based superconductors. Reprinted with permission from Ref. [48] (Copyright 2010, IOP Publishing). (c) Anion height-dependence of $T_{\mathrm{c}}$ for FeSe-derived superconductors [41,42].

der from FeP, FeAs, FeSe, to FeTe. For the FeAs-based 1111 phase, as the substitutions of $\mathrm{Nd}$ and $\mathrm{Sm}$ for La increase $h$ to approximately $1.38 \AA, T_{c}$ increases abruptly from 26 to $55 \mathrm{~K}$, which is the maximum bulk value among all the Fe-based superconductors. FeP-based superconductors have relatively small $h$ (compared with the optimal $1.38 \AA$ ) and, as a result, their $T_{c}$ values are significantly lower than those in FeAs-based superconductors. For example, in the La-1111 phase, as $\mathrm{P}$ is replaced by As, $T_{c}$ is dramatically enhanced from 7 to $26 \mathrm{~K}$ and accompanied by increasing $h$. After crossing this maximum, the $T_{\mathrm{c}}$ of $\mathrm{TbFeAsO}_{0.7}, \mathrm{Ba}_{0.6} \mathrm{~K}_{0.4}$ $\mathrm{Fe}_{2} \mathrm{As}_{2}, \mathrm{NaFeAs}$, and LiFeAs decrease in order of further increases in $h$. The data obtained for optimally doped FeSe ${ }_{1-x}$ $\mathrm{Te}_{x}$ and $\mathrm{FeSe}_{0.57} \mathrm{Te}_{0.43}$ seem to follow the same trend. As a result, this $h$-dependence of $T_{c}$ seems to be universal for $1111,122,111$, and 11 iron-based superconductors. Although the maximum $T_{c}$ of superconductors with a thick blocking layer remains unconfirmed, the data obtained for the 42622 superconductor obey the same universal curve, apart from a small deviation, which may suggest that the enhancement of the two-dimensional characteristic could induce a $T_{\mathrm{c}}$ greater than $56 \mathrm{~K}$. Although such a universal relationship exists between $h$ and $T_{c}$, an exception does, in fact, exist in the case of FeSe-derived superconductors. As shown in Fig. 2c, for FeSe-derived materials, a minimum $T_{\mathrm{c}}$ value (instead of a maximum) can be observed at $h \approx$ $1.45 \AA[41,42]$, as shown in Fig. 2b. This may suggest the existence of some new, currently unknown, underlying physics in FeSe-derived superconductors compared with that of FeAs-based materials.

\section{PHASE DIAGRAMS}

Chemical doping or external/chemical pressure has been applied to the parent compounds of iron-based superconductors in order to obtain superconductivity. Investigation of the relevant phase diagram is very helpful for under- standing the mechanism of this superconductivity. Hence, one can clearly see how the superconductivity emerges in response to chemical doping or external pressure (see Fig. 3) [50-58]. In general, the parent compound of an ironbased superconductor is an antiferromagnetic (AFM) bad metal, as shown in Fig. 4 [59,60]. Taking into account the fact that the parent compounds are usually poor metals, the mechanism of this AFM was historically ascribed to the spin-density-wave (SDW) ordering of itinerant electrons $[59,61]$. In contrast, the AFM in cuprate parent compounds arises from the superexchange of local moments [62]. The applicability of the itinerant electron model to pnictides was strongly supported by earlier angle-resolved photoemission spectroscopy (ARPES) experiments [63] and the first-principle calculations [64-67], which indicated that the Fermi surfaces of the parent compounds have perfect Fermi nesting conditions. However, in subsequent experiments, the simple itinerant electron model was found to provide an incomplete description, and a model with coexisting itinerant electrons and local moments should therefore be considered in order to obtain a comprehensive understanding of AFM in iron-based superconductors [68].

The parent compounds of iron-based superconductors have magnetic structures, as shown in Figs $4 \mathrm{a}$ and $4 \mathrm{~b}$. Neutron diffraction experiments have revealed that the magnetic wave vector in $\mathrm{LaOFeAs}$ is $(1 / 2,1 / 2,1 / 2)_{\mathrm{T}}=(1,0$, $1 / 2)_{\mathrm{O}}$, where $\mathrm{T}$ and $\mathrm{O}$ indicate tetragonal and orthorhombic phases, respectively, and the magnetic moment per Fe is $0.36 \mu_{\mathrm{B}}$. In LaOFeAs, the in-plane magnetic spins of the Fe atoms are arranged as shown in Fig. 4a. The spins lie within the $a b$ plane, and are aligned antiferromagnetically along the orthorhombic $a$ axis and ferromagnetically along the orthorhombic $b$ axis $[60,61,69]$. The in-plane magnetic alignment for all the parent compounds of FeAs-based superconductors $(111,122$, and 1111 systems $)$ is similar to that of LaOFeAs. While the interplane spin alignment de- 

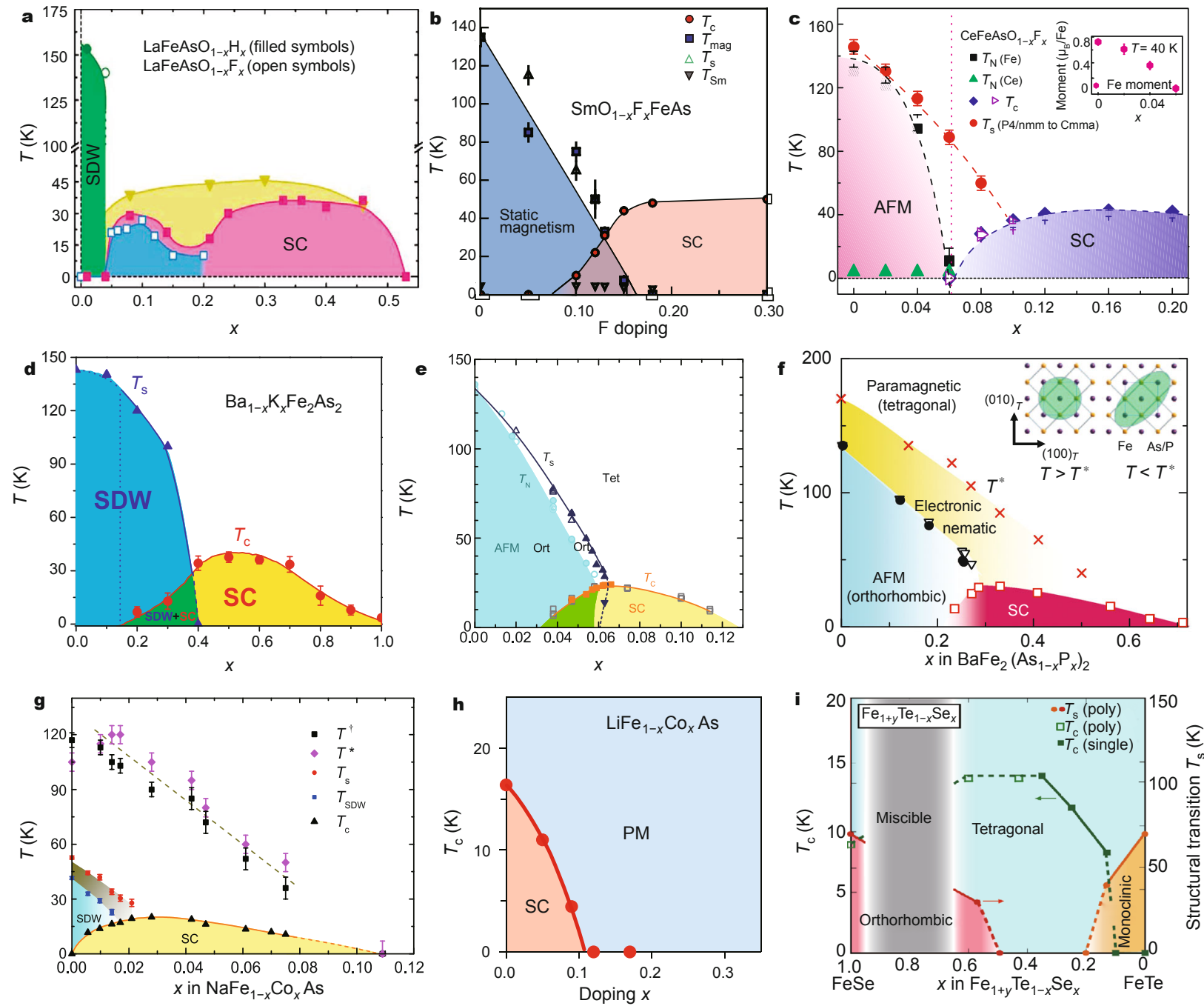

Figure 3 Typical electronic and magnetic phase diagrams of several series of iron-based superconducting systems [50-58]. Reprinted with permission from (a) Ref. [50] (Copyright 2012, Nature Publishing Group), (b) Ref. [51] (Copyright 2009, Nature Publishing Group), (c) Ref. [52] (Copyright 2008, Nature Publishing Group), (d) Ref. [53] (Copyright 2009, IOP Publishing), (e) Ref. [54] (Copyright 2010, American Physical Society), (f) Ref. [55] (Copyright 2012, Nature Publishing Group), (g) Ref. [56], (h) Ref. [57], and (i) Ref. [58] (Copyright 2010, the Physical Society of Japan).

pends on the system, the spins in $\mathrm{LaOFeAs}, \mathrm{NdOFeAs}$, and $\mathrm{BaFe}_{2} \mathrm{As}_{2}$, for instance, are antiferromagnetically aligned along the $c$ axis, while those in $\mathrm{CeOFeAs}$ and PrOFeAs are ferromagnetically aligned along the $c$ axis [60]. $\mathrm{Fe}_{1+x} \mathrm{Te}$ exhibits a very different alignment formation from that seen in FeAs-based compounds, and has quite complicated magnetic structures. In the AFM state, $\mathrm{Fe}_{1+x}$ Te has a magnetic wave vector $(1 / 2,0,1 / 2)$ (see Fig. $4 b)$ [70,71], that is, the spins align in a dual-AFM stripe in the crystallographic direction with a magnetic moment of $2.03 \mu_{\mathrm{B}}$ per Fe atom (for $x=0.076$ ) [71]. It is worth noting that the easy axis of the magnetic order in $\mathrm{A}_{x} \mathrm{Fe}_{2-y} \mathrm{Se}_{2}$ superconductors is the $c$-axis [72], which is distinct from the in-plane magneticordered alignment of spins in FeAs-based compounds. At present, it is widely believed that the AFM order with very high transition temperature $(\sim 500 \mathrm{~K})$ and large moment $\left(\sim 3.3 \mu_{\mathrm{B}} / \mathrm{Fe}\right)$ in $\mathrm{A}_{x} \mathrm{Fe}_{2-y} \mathrm{Se}_{2}$ is phase-separated from the superconductivity.

Through various types of chemical doping or the application of external pressure, the AFM order is suppressed and the ground states can be effectively tuned from AFM to superconducting phases. They then exhibit a quite universal phase diagram (see Fig. 3), which is similar to that of cuprate superconductors. This may suggest a possible common mechanism in both high- $T_{\mathrm{c}}$ superconductor families. In fact, with cooling from room temperature, the parent compounds first exhibit an interesting structural transition from a high-temperature tetragonal structure to a low-tem- 

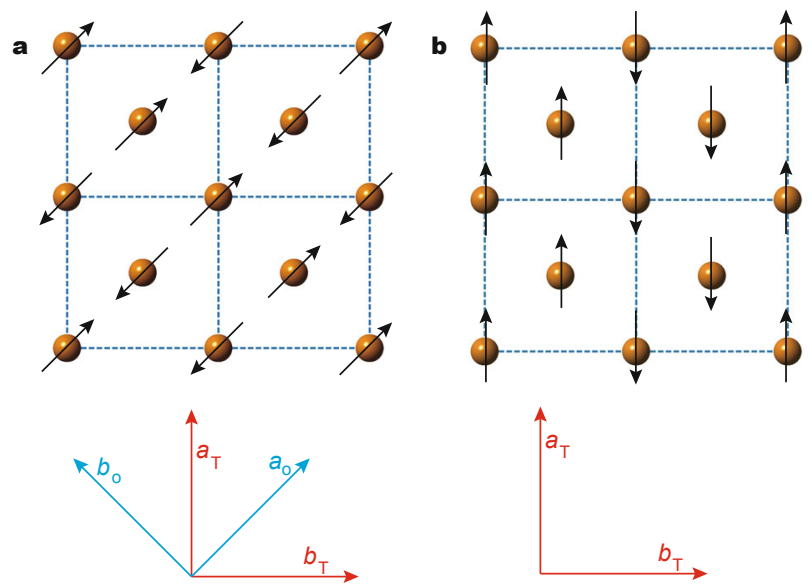

Figure 4 (a) In-plane spin alignment in the 111, 122, and $1111 \mathrm{FeAs}-$ based systems $[60,69]$. (b) In-plane spin alignment in the 11 system $[70,71]$.

perature orthorhombic structure, followed by the AFM transition [59]. The structural transition temperature $\left(T_{\mathrm{s}}\right)$ is usually slightly higher than or equal to the AFM transition temperature $\left(T_{\mathrm{N}}\right)$ (see Fig. 3). Obvious anomalies associated with the structural and magnetic transitions can be observed in the transport measurements, as shown in Fig. $5[73,74]$. Such structural transition is ascribed to electron-driven phase transition rather than pure structural effects, and strongly couples to the subsequent AFM transition [75-78]. The underlying mechanism of the structural transition is also beyond the capacity of the simple itinerant electron model.

By tuning doping with different chemicals to a moderate level, superconductivity can be achieved for either hole- or electron-type carrier cases. As shown in Fig. 3, both structural and AFM transitions are suppressed continuously by doping with either hole- and electron-type carriers. Meanwhile, superconductivity emerges above the critical doping level and may coexist with the suppressed AFM order in part of the phase diagram. At first, $T_{c}$ is enhanced with increased doping levels. It then reaches a maximum at the so-called "optimal doping level" and finally decreases to zero with further increased doping. The highest $T_{\mathrm{c}}$ in bulk materials, up to $55 \mathrm{~K}$, was obtained for F-doped SmOFeAs $[4,5,33]$, while values of $T_{\mathrm{c}}$ of up to $\sim 100 \mathrm{~K}$ have been reported in single-layer FeSe film [79-83]. Typically, the superconducting region below the optimal doping level in the phase diagram is defined as the "underdoped superconducting region". This is the same term as that used for cuprates. The area above the optimal doping level, where $T_{c}$ is suppressed, is called the "overdoped superconducting region". The entire superconducting region has a dome-like shape in the phase diagram.

One of the most important issues in iron-based superconductivity is the coexistence of the superconductivity with the AFM/SDW order. In F-doped LaOFeAs [84], PrOFeAs [85], and NdOFeAs [86], as shown in Figs 3a and $3 \mathrm{~b}$, the structural and AFM/SDW transitions vanish
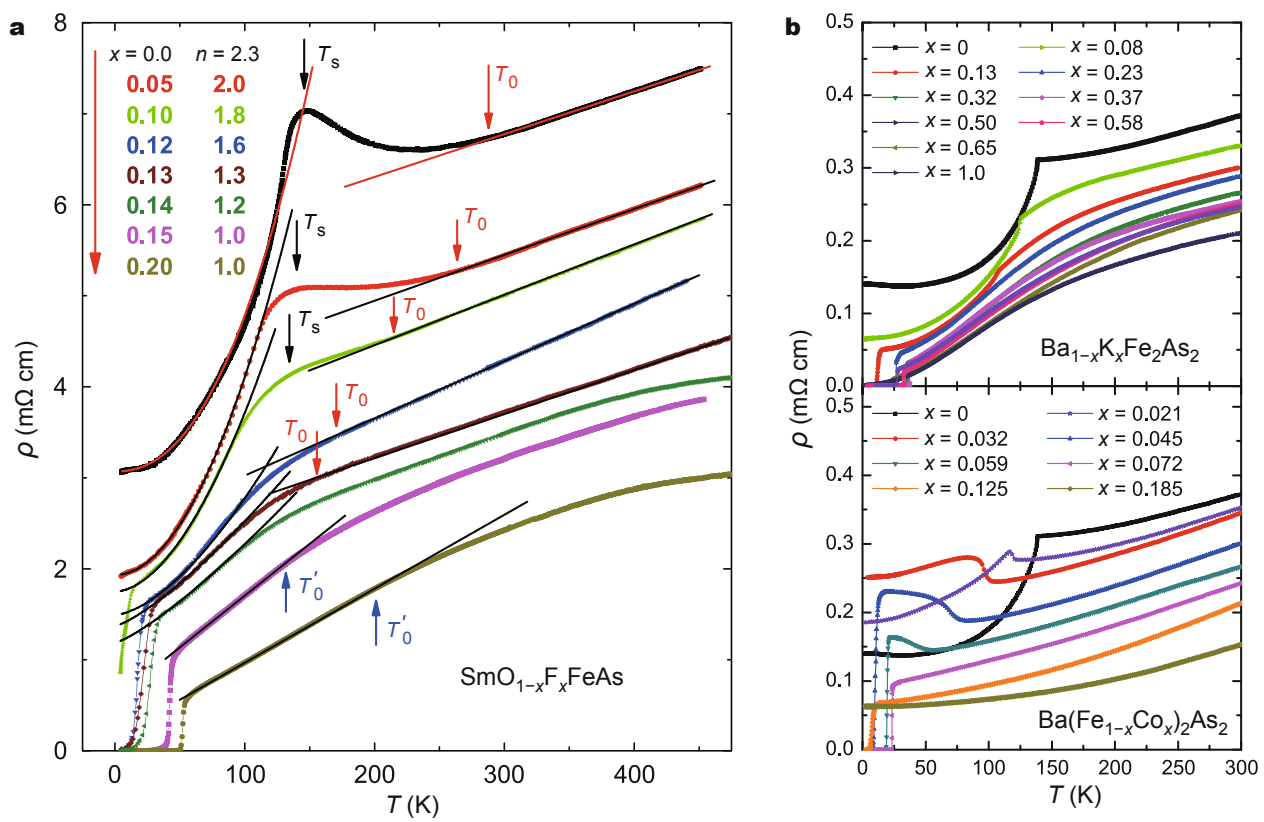

Figure 5 Temperature dependence of resistivity for the: (a) 1111 system (polycrystalline $\mathrm{SmO}_{1-x} \mathrm{~F}_{x} \mathrm{FeAs}$ ) [Reprinted with permission from Ref. [73] (Copyright 2008, American Physical Society)]; (b) 122 system (hole-doped $\mathrm{Ba}_{1-x} \mathrm{~K}_{x} \mathrm{Fe}_{2} \mathrm{As}_{2}$ and electron-doped $\mathrm{Ba}$ (Fe ${ }_{1-x} \mathrm{Co}_{x}$ ) $\mathrm{As}_{2}$ single crystals). Reprinted with permission from Ref. [74]. 
abruptly at a certain doping level, exhibiting a step-like behavior, and the superconductivity then emerges. There is no coexistence region for AFM/SDW ordering and superconductivity in the phase diagrams of these 1111 materials. In F-doped CeOFeAs [52], the structural and AFM/ SDW transitions disappear continuously, but there is also no coexistence between the AFM/SDW ordering and superconductivity; the superconductivity simply appears when the AFM/SDW ordering is completely suppressed. These phases seem to be connected by a quantum critical point (QCP). In F-doped SmOFeAs [51], the structural and AFM/SDW transitions also go to zero continuously, but a region exhibiting coexistence between the AFM/SDW ordering and superconductivity exists. This suggests that the destruction of the long-range magnetic order is not an essential condition for the emergence of superconductivity. Such a coexistence of AFM/SDW ordering and superconductivity has been widely observed in 122 and 111 materials $[14,15,53,54]$, but not in 1111 materials. The phase diagram of the 1111 system should therefore be re-examined in order to determine whether the coexistence of SDW and superconductivity occurs in LaOFeAs and CeOFeAs systems.

For the doped 122 materials, the question of whether the AFM/SDW and superconducting states coexist microscopically or are phase separated has received considerable attention. For hole doping with K, early studies on powder samples using techniques such as ${ }^{75} \mathrm{As}$ nuclear magnetic resonance $(\mathrm{NMR})$ [87], muno-spin rotation ( $\mu \mathrm{SR})$ [88], and magnetic force microscopy (MFM) [88] consistently indicated the existence of both magnetically ordered and nonmagnetic regions, as expected for microscopic phase separation. Analyses of microstrains measured using X-ray, neutron diffraction and, later, ${ }^{75} \mathrm{As}$ NMR techniques on single crystals also supported the occurrence of electronic phase separation. In contrast, ${ }^{57} \mathrm{Fe}$-Mössbauer measurements detected complete magnetic ordering in a $\mathrm{K}$-doped $\mathrm{BaFe}_{2} \mathrm{As}_{2}$ sample, consistent with that expected based on the microscopic coexistence of the AFM/SDW and superconducting states [89]. Recently, Li et al. [90] presented unambiguous ${ }^{75}$ As NMR evidence that AFM/SDW ordering and superconductivity coexist microscopically in the case of high-quality underdoped $\mathrm{Ba}_{1-x} \mathrm{~K}_{x} \mathrm{Fe}_{2} \mathrm{As}_{2}$ single crystals. Considering the sample quality in the earlier studies, the microscopic coexistence of AFM/SDW ordering and superconductivity is most likely an intrinsic phenomenon in $\mathrm{K}$-doped $\mathrm{BaFe}_{2} \mathrm{As}_{2}$. In addition, for Co-doped 122 samples, both ${ }^{75}$ As NMR [91] and $\mu$ SR measurements [92] have also indicated the microscopic coexistence of superconductivity and AFM/SDW ordering. Further, in isovalently doped 122 materials (P-doped $\mathrm{BaFe}_{2} \mathrm{As}_{2}$ and Ru-doped $\mathrm{BaFe}_{2} \mathrm{As}_{2}$ ), the microscopic coexistence of superconductivity and AFM/
SDW ordering has been confirmed by NMR experiments [93]. As a result, it can be concluded that the microscopic coexistence of superconductivity and AFM/SDW ordering is universal in FeAs-122 systems. Furthermore, neutron diffraction measurements conducted on Co-doped samples $(x=0.04$ and 0.047$)[94,95]$ have indicated that the magnetic Bragg peak intensity of the AFM/SDW state is suppressed when entering the superconducting state, suggesting a very strong interaction between the superconductivity and SDW ordering. Such suppression can be attributed to the same electrons participating in both the SDW and superconductivity, so that the phase coexistence scenario is favored.

The evolution of finite-temperature electronic behavior with varying doping levels is also interesting and has been studied intensively. F-doped SmOFeAs can be taken as an example to illustrate the evolution of finite-temperature electronic behavior with F content, as shown in Fig. 5a [73]. Here, the low-temperature resistivity can be well fitted against $a+b T^{n}$, and the fitting parameter, $n$, shows a systematical change from 2.3 to 1 with increasing F content, from $x=0$ to 0.15 . It is intriguing that the temperature dependence of the low-temperature resistivity just above $T_{\mathrm{c}}$ changes to $T$-linear dependence with an increase in $\mathrm{F}$ content from $x=0.14$ to 0.15 , suggesting that a QCP appears in the region of $x=0.14$. This occurs at the same time as the suppression of the AFM/SDW order. Such evolution of the finite-temperature electronic behavior has also been widely observed in other iron-based superconductors, such as the P-doped $\mathrm{BaFe}_{2} \mathrm{As}_{2}$ system [96,97]. Particularly, the scattering of charge carriers by fluctuation associated with the QCP, which is widely used to explain the $T$-linear resistivity in heavy-fermion metals [98], has been considered as a possible explanation for the $T$-linear resistivity observed in iron-based superconductors.

Besides the structural and magnetic transitions, an unexpected in-plane electronic anisotropy begins to emerge at temperatures well above $T_{s}$ (as shown in Figs $3 \mathrm{f}$ and $3 \mathrm{~g}$ ). This is indicated by the resistivity, reflectivity, and ARPES measurements of detwinned single crystals of underdoped 122 materials [98]. As shown in Fig. 6a [99,100], this inplane electronic anisotropy appears for a tetragonal structure at temperatures well above $T_{s}$, with a new temperature scale $\left(T^{*}\right)$ (as marked in Figs $3 f$ and $3 g$ ) $[55,56]$. This anisotropy has been attributed to the formation of electronic nematicity, a unidirectional self-organized state that breaks the rotational symmetry of the underlying lattice [99]. On the hole-doped side, the in-plane electronic anisotropy decreases very rapidly and has extremely small values. It can even exhibit sign reversal in anisotropy of in-plane resitivity (sign of $\rho_{\mathrm{b}}-\rho_{\mathrm{a}}$ ) at some relatively high doping levels [101104]. In sharp contrast, the in-plane electronic anisotropy 

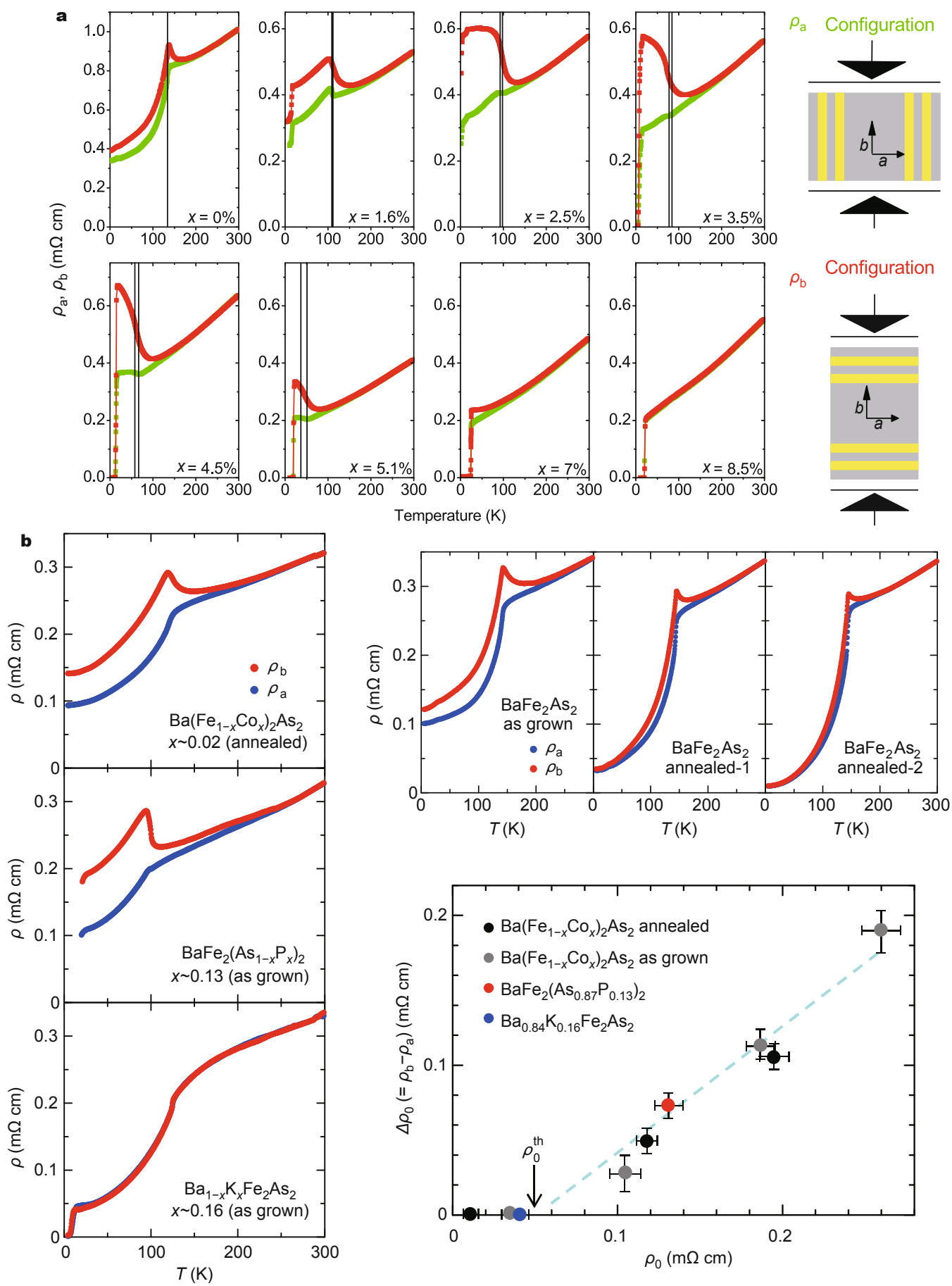

Figure 6 (a) Anisotropic in-plane resistivity in $\mathrm{Ba}\left(\mathrm{Fe}_{1-x} \mathrm{Co}_{x}\right)_{2} \mathrm{As}_{2}$ single crystals. Reprinted with permission from Ref. [100] (Copyright 2010, American Association for the Advancement of Science). (b) Studies on the effect of impurity scattering on in-plane anisotropy in doped $\mathrm{BaFe}_{2} \mathrm{As}_{2}$ single crystals. Reprinted with permission from Ref. [102] (Copyright 2013, American Chemical Society).

was first enhanced on the electron-doped side and gradually disappeared with further doping $[99,100]$. The electronic nematicity revises our understanding of the phase diagram of normal states, but its origin has been quite controversial.
Recently, annealing experiments (see Fig. 6b) and scanning tunneling microscope (STM) results have revealed that the in-plane electronic anisotropy is most likely triggered by dopant atoms $[102,105,106]$. STM analysis of Co-doped 
$\mathrm{CaFe}_{2} \mathrm{As}_{2}$ has revealed that substituting $\mathrm{Co}$ for $\mathrm{Fe}$ atoms generates a dense population of identical anisotropic impurity states. These impurity states then scatter quasiparticles in a highly anisotropic manner, suggesting that anisotropic scattering by dopant-induced impurity states is the source of the electronic nematicity [106]. However, determining whether this model is applicable to all 122 materials that exhibit electronic nematicity requires further investigation.

The iron chalcogenide, $\mathrm{Fe}_{1+y}\left(\mathrm{Te}_{1-x} \mathrm{Se}_{x}\right)$, has the simplest structure of the iron-based superconductors $[6,9,10]$. Although its Fermi surface is similar to that of iron pnictides $[107,108]$, the parent compound, $\mathrm{Fe}_{1+y} \mathrm{Te}$, exhibits an AFM order with an in-plane magnetic wave vector $(\pi, 0)$. This is in contrast to the parent compounds of iron pnictides, in which the magnetic order has an in-plane magnetic wave vector, $(\pi, \pi)$, that connects the hole and electron parts of the Fermi surface. Moreover, as mentioned above, the spin alignment configuration in the magnetically ordered state in $\mathrm{Fe}_{1+y} \mathrm{Te}$ is distinct from that in iron-pnictide parent compounds. For $\mathrm{Fe}_{1.141} \mathrm{Te}$, a tetragonal-orthorhombic structural transition and an incommensurate magnetic wave vector, $q$, of $( \pm \delta, 0,1 / 2)$ have been reported [71]. With decreasing excess Fe concentration, the incommensurate magnetic wave vector transitions continuously change to a commensurate magnetic wave in $\mathrm{Fe}_{1.076} \mathrm{Te}$. Furthermore, the superconductivity tends to be suppressed with greater excess Fe content $[109,110]$. The phase diagram of $\mathrm{Fe}_{1+y} \mathrm{Te}_{1-x}$ $\mathrm{Se}_{x}$, with a low excess Fe concentration, is shown in Fig. $3 \mathrm{i}$ [58]. As can be seen in this figure, the structural transition from the high-temperature tetragonal phase to the low-temperature monoclinic phase in $\mathrm{Fe}_{1+y} \mathrm{Te}$ occurs at the same temperature as the AFM transition. At the other side of the $\mathrm{Fe}_{1+y}\left(\mathrm{Te}_{1-x} \mathrm{Se}_{x}\right)$ phase diagram, $\mathrm{FeSe}_{1-x}$ exhibits superconductivity below $10 \mathrm{~K}$. A structural transition also occurs in this material, from the high-temperature tetragonal to the low-temperature orthorhombic phase, but without any magnetic order following [111], which is in contrast to other iron-based superconductors. The tetragonal-orthorhombic structural transition observed in FeSe is suppressed with increasing Te concentration, and the highest $T_{\mathrm{c}}$ appears in the tetragonal phase near $x<0.5$. With a further increase in Te content, the value of $T_{\mathrm{c}}$ reduces, the AFM ordering accompanying the tetragonal-monoclinic distortion appears, and the bulk superconductivity disappears. As shown in Fig. 3i, a miscible region $(A+B)$ exists at approximately $x=0.7$ to 0.95 , at which phase separations occur.

As mentioned above, external pressure can also be used to tune the magnetism and superconductivity. It can be seen in Fig. 7a [112] that, in the majority of iron-pnictide systems, the application of pressure can enhance $T_{c}$ efficiently, provided the pressure is below a moderate value. The value of $T_{\mathrm{c}}$ can then be suppressed as the pressure increases further, for example, in doped LaFeAsO [113], underdoped 122 systems [114], and doped NaFeAs [16,115,116]. In particular, superconductivity can be induced by pressure in non-superconducting $\mathrm{LaOFeAs}$ and $\mathrm{AeFe}_{2} \mathrm{As}_{2}$ ( $\mathrm{Ae}=$ $\mathrm{Ca}, \mathrm{Sr}, \mathrm{Ba}, \mathrm{Eu}$ ), which is accompanied by the suppression of the AFM/SDW transition in these systems [117,118]. In fact, suppression of the AFM/SDW transition by pressure can be observed for all the underdoped samples, as shown in Fig. 7b, provided a magnetic transition exists. Fig. $7 \mathrm{~b}$ also demonstrates that pressure can expand the superconducting region to a lower doping level [119]. It should be noted that external or chemical pressure has an obvious effect on the magnetism in the FeAs or FeSe layer, but has a negligible influence on the magnetic order located outside these layers. For example, the external pressure does not change the AFM transition temperature of $\mathrm{Eu}^{2+}$ ions in $\mathrm{EuFe}_{2} \mathrm{As}_{2}$ and doped $\mathrm{EuFe}_{2} \mathrm{As}_{2}[118,120]$. Moreover, the chemical pressure produced by $\mathrm{S}$ substituted in place of $\mathrm{Se}$ in $\mathrm{Li}_{0.8} \mathrm{Fe}_{0.2} \mathrm{OHFeSe}$ has no effect on the AFM transition in the $\mathrm{Li}_{0.8} \mathrm{Fe}_{0.2} \mathrm{OH}$ layer [121]. In FeSe, as shown in Fig. $7 \mathrm{~d}, T_{\mathrm{c}}$ can also be improved from $8 \mathrm{~K}$ at ambient pressure to $37 \mathrm{~K}$ at approximately $9 \mathrm{GPa}$, but then decreases with further increase in pressure [7]. In some compositions of $\mathrm{K}_{1-x} \mathrm{Fe}_{y} \mathrm{Se}_{2}$ and $\mathrm{Cs}_{1-x} \mathrm{Fe}_{y} \mathrm{Se}_{2}$ crystals, this hump-shaped pressure dependence of $T_{c}$ can also be observed [122]. More generally, however, the suppression of $T_{c}$ by pressure can be seen in $\mathrm{K}_{1-x} \mathrm{Fe}_{y} \mathrm{Se}_{2}$ samples [122-124], where $T_{\mathrm{c}}$ goes to zero at approximately $10 \mathrm{GPa}[123,124]$. The most intriguing aspect of $\mathrm{K}_{1-x} \mathrm{Fe}_{y} \mathrm{Se}_{2}$ is that a new superconducting state emerges with further increases in pressure, exhibiting a significantly higher $T_{\mathrm{c}}(\sim 48 \mathrm{~K}$ at approximately $12 \mathrm{GPa})$, as shown in Fig. 7e [123]. It is apparent that the pressure dependence of $T_{\mathrm{c}}$ actually depends on the detailed materials. For example, in contrast to the behavior observed in doped LaOFeAs, monotonic suppression of $T_{c}$ can be obtained in doped NdOFeAs and SmOFeAs systems, as shown in Fig. 7a [112].

The contrasting pressure-dependence behavior of $T_{c}$ among different systems was previously thought to be related to the specific crystallographic detail of each substance. As discussed above, this can be reflected by an empirically inverse $\mathrm{V}$-shaped dependence of $T_{\mathrm{c}}$ on anion height from the Fe layer in iron-pnictide compounds, with the optimal $T_{\mathrm{c}}$ occurring at $h_{0} \gg 1.38 \AA$ [48]. For example, doped LaOFeAs samples are located on the side of the diagram having values of $h$ that are significantly smaller than $h_{0}$, and external pressure causes these systems to shift to the area close to $h_{0}$ [125]. This results in a dramatic increase in $T_{c}$. However, for doped SmOFeAs and NdOFeAs with $T_{c}$ of approximately $55 \mathrm{~K}$, the anion heights from the Fe layer are almost equal to $h_{0}$ [48]; thus, any applied pressure will suppress $T_{c}$, because this pressure will separate the anion height 

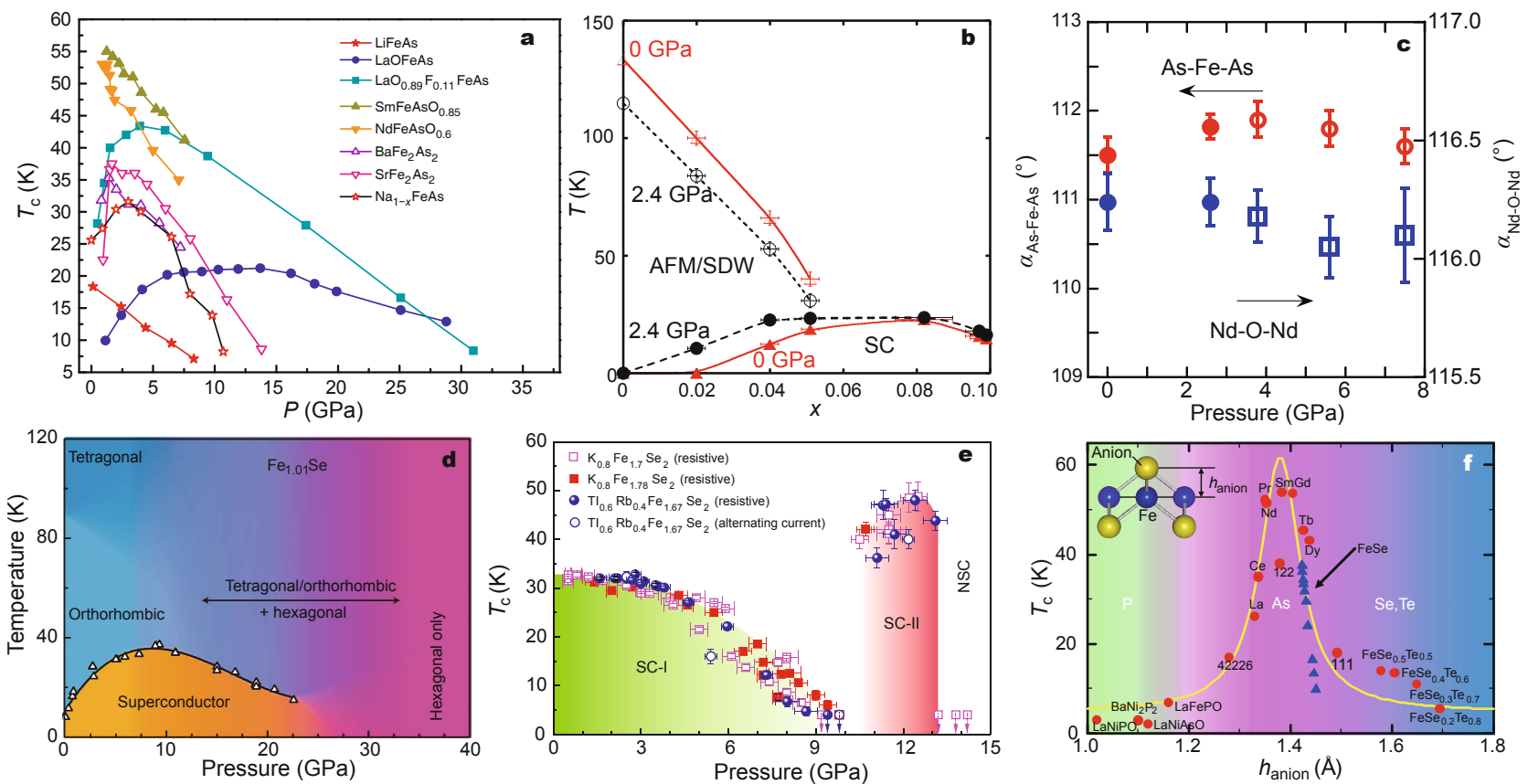

Figure 7 Phase diagrams under external pressure. (a) External-pressure dependence of $T_{\mathrm{c}}$ for several typical iron-pnictide compounds [112,115]. Reprinted with permission from Ref. [112] (Copyright 2011, American Physical Society) and [115] (Copyright 2009, IOP Publishing). (b) Dopingdependent phase diagram of $\mathrm{Ba}\left(\mathrm{Fe}_{1-x} \mathrm{Co}_{x}\right)_{2} \mathrm{As}_{2}$ under an applied pressure of 0 and $2.4 \mathrm{GPa}$. Reprinted with permission from Ref. [119] (Copyright 2009, American Physical Society). (c) Evolution of As-Fe-As $\alpha$-value for $\mathrm{NdO}_{0.85} \mathrm{Fe} A s\left(T_{\mathrm{c}}=51 \mathrm{~K}\right.$ at ambient pressure) under external pressure (red circles). Reprinted with permission from Ref. [128] (Copyright 2009, the Physical Society of Japan). (d) External-pressure-dependent phase diagram of $\mathrm{Fe}_{1.01} \mathrm{Se}$. Reprinted with permission from Ref. [7] (Copyright 2009, Nature Publishing Group). (e) External-pressure dependence of $T_{\mathrm{c}}$ for $\mathrm{K}_{0.8} \mathrm{Fe}_{1.7} \mathrm{Se} \mathrm{e}_{2}$, $\mathrm{K}_{0.8} \mathrm{Fe}_{1.78} \mathrm{Se}_{2}$, and $\mathrm{Tl}_{0.6} \mathrm{Cs}_{0.4} \mathrm{Fe}_{1.67} \mathrm{Se}_{2}$. Reprinted with permission from Ref. [123] (Copyright 2012, Nature Publishing Group). (f) Anion height dependence of $T_{\mathrm{c}}$ under external pressure for FeSe samples (blue triangular circles). Reprinted with permission from Ref. [126] (Copyright 2010, American Physical Society).

of the system from $h_{0}$. The pressure dependence of $T_{c}$ in FeSe also seems to exhibit a very close relationship with the anion height from the Fe layer [126], as shown in Fig. $7 f$. However, from our study incorporating other FeSe-derived superconductors, as mentioned above, the anion height dependence of $T_{c}$ in FeSe-derived systems may have a shape that is distinct from that obtained for iron-pnictide superconductors, as can be seen in Fig. 3c. The maximum value of $T_{c}$ was thought to be clearly obtained in iron-pnictide compounds, when the $\mathrm{FeX}_{4}$ tetrahedron achieved a regular shape [127]. However, the correlation between $T_{c}$ and the Se-Fe-Se $\alpha$-value discussed above has suggested that a regular $\mathrm{FeSe}_{4}$ tetrahedron may not be a prerequisite for higher $T_{c}$ [42]. In fact, the results of pressure studies on $\mathrm{NdO}_{0.85} \mathrm{FeAs}$ also imply that the regularity of the $\mathrm{FeAs}_{4}$ tetrahedron may have less correlation to $T_{c}$ than the anion height. Here, the As-Fe-As $\alpha$-value changes very slightly (see Fig. 7c), in spite of the large decrease in $T_{\mathrm{c}}$ from 51 to $36 \mathrm{~K}$ in response to a change in pressure from ambient to $8 \mathrm{GPa}$ [128].

Despite certain differences in the doping-dependent phase diagrams of the various iron-based superconductors, a close inspection of Fig. 3 indicates that some common features exist. For example, all systems exhibit an AFM/ SDW state for the parent compounds, which is suppressed with doping, while superconductivity is induced with further doping. A strong similarity to the generic phase diagram of cuprates is suggested, which provides evidence for the interplay between magnetism and superconductivity in Fe-based materials.

In summary, we have briefly reviewed the crystal structure features of all iron-based superconductors. It is notable that the superconductivity is not, in fact, directly related to the thickness of the spacer slabs between adjacent conducting FeAs/FeSe layers. This is in sharp contrast to the strong dependence of the superconductivity in cuprates on the distance between the $\mathrm{CuO}_{2}$ planes. Instead, the superconductivity in iron-based superconductors has obvious dependence on the crystallographic parameters (such as the height of the anion from the Fe layer or the anion-Fe-anion angle). It should be noted that this indicates that the mechanism of superconductivity in iron-based superconductors is somewhat different from that in cuprates, although considerable effort has been expended in attempting to 
unify the origins of the high-temperature superconductivity exhibited by these two superconducting families. However, despite certain subtle differences between the phase diagrams of the various iron-based superconductor systems, some common features that are extremely similar to cuprate characteristics have been observed, which causes researchers to believe that the interplay between magnetism and superconductivity plays a crucial role in both high-temperature superconducting families. On the other hand, the mechanism causing the coexistence of the AFM/ SDW order and superconductivity, along with the nature of the notable electronic anisotropy (possibly nematicity) in the underdoped region, which may possibly be closely related to the occurrence of superconductivity in iron-based superconductors, remain unresolved at present. It appears that significantly more research is required, and it is hoped that this review will provide some useful clues to aid further investigation of these topics.

Received 26 December 2014; accepted 8 January 2015; published online 23 January 2015

1 Kamihara $\mathrm{Y}$, Watanabe T, Hirano $\mathrm{M}$, et al. Iron-based layered superconductor $\mathrm{La}\left[\mathrm{O}_{1-x} \mathrm{~F}_{x}\right] \mathrm{FeAs}(x=0.05-0.12)$ with $T_{\mathrm{c}}=26 \mathrm{~K}$. J Am Chem Soc, 2008, 130: 3296-3297

2 Chen XH, Wu T, Wu G, et al. Superconductivity at $43 \mathrm{~K}$ in SmFeAs $\mathrm{O}_{1-x} \mathrm{~F}_{x}$. Nature, 2008, 453: 761-762

3 Kamihara Y, Hiramatsu H, Hirano M, et al. Iron-based layered superconductor: LaOFeP. J Am Chem Soc, 2006, 128: 10012-10013

4 Ren ZA, Lu W, Yang J, et al. Superconductivity at $55 \mathrm{~K}$ in iron-based F-doped layered quaternary compound $\mathrm{Sm}\left[\mathrm{O}_{1-x} \mathrm{~F}_{x}\right]$ FeAs. Chin Phys Lett, 2008, 25: 2215-2216

5 Ren ZA, Che GC, Dong XL, et al. Superconductivity and phase diagram in iron-based arsenic-oxides $\mathrm{ReFeAsO}_{1-\delta}(\mathrm{Re}=$ rare earth metal) without fluorine doping. EPL, 2008, 83: 17002

6 Hsu FC, Luo JY, Yeh KW, et al. Superconductivity in the PbO-type structure a-FeSe. Proc Natl Acad Sci USA, 2008, 105: 14262-14264

7 Medvedev S, McQueen TM, Troyan IA, et al. Electronic and magnetic phase diagram of $\beta-\mathrm{Fe}_{1.01}$ Se with superconductivity at $36.7 \mathrm{~K}$ under pressure. Nat Mater, 2009, 8: 630-633

8 Margadonna S, Takabayashi Y, Ohishi Y, et al. Pressure evolution of the low-temperature crystal structure and bonding of the superconductor FeSe $\left(T_{\mathrm{c}}=37 \mathrm{~K}\right)$. Phys Rev B, 2009, 80: 064506

9 Yeh KW, Huang TW, Huang YL, et al. Tellurium substitution effect on superconductivity of the $\alpha$-phase iron selenide. EPL, 2008, 84: 37002

10 Sales BC, Sefat AS, McGuire MA, et al. Bulk superconductivity at $14 \mathrm{~K}$ in single crystals of $\mathrm{Fe}_{1+y} \mathrm{Te}_{x} \mathrm{Se}_{1-x}$. Phys Rev B, 2009, 79: 094521

11 Deng Z, Wang XC, Liu QQ, et al. A new 111 type iron pnictide superconductor LiFeP. EPL, 2009, 87: 3704

12 Wang XC, Liu QQ, Lv YX, et al. The superconductivity at $18 \mathrm{~K}$ in LiFeAs system. Solid State Commun, 2008, 148: 538-540

13 Parker DR, Pitcher MJ, Baker PJ, et al. Structure, antiferromagnetism and superconductivity of the layered iron arsenide NaFeAs. Chem Commun, 2009, 2189-2191

14 Parker DR, Smith MJP, Lancaster T, et al. Control of the competition between a magnetic phase and a superconducting phase in cobalt-doped and nickel-doped NaFeAs using electron count. Phys Rev Lett, 2010,104: 057007

15 Wang AF, Luo XG, Yan YJ, et al. Phase diagram and calorimetric properties of $\mathrm{NaFe}_{1-x} \mathrm{Co}_{x}$ As. Phys Rev B, 2012, 85: 224521

16 Wang AF, Lin JJ, Cheng $\mathrm{P}$, et al. Phase diagram and physical properties of $\mathrm{NaFe}_{1-x} \mathrm{Cu}_{x}$ As single crystals. Phys Rev B, 2013, 88: 094516

17 Rotter M, Tegel M, Johrendt D. Superconductivity at $38 \mathrm{~K}$ in the Iron Arsenide $\left(\mathrm{Ba}_{1-x} \mathrm{~K}_{x}\right) \mathrm{Fe}_{2} \mathrm{As}_{2}$. Phys Rev Lett, 2008, 101: 107006

$18 \mathrm{Wu} \mathrm{G}$, Chen $\mathrm{H}, \mathrm{Wu} \mathrm{T}$, et al. Different resistivity response to spin density wave and superconductivity at $20 \mathrm{~K}$ in $\mathrm{Ca}_{1-x} \mathrm{Na}_{x} \mathrm{Fe}_{2} \mathrm{As}_{2}$. J Phys Condens Matter, 2008, 20: 422201

19 Ronning F, Klimczuk T, Bauer ED, et al. Synthesis and properties of $\mathrm{CaFe}_{2} \mathrm{As}_{2}$ single crystals. J Phys Condens Matter, 2008, 20: 322201

20 Sasmal K, Lv B, Lorenz B, et al. Superconducting Fe-based compounds $\left(\mathrm{A}_{1-x} \mathrm{Sr}_{x}\right) \mathrm{Fe}_{2} \mathrm{As}_{2}$ with $\mathrm{A}=\mathrm{K}$ and $\mathrm{Cs}$ with transition temperatures up to $37 \mathrm{~K}$. Phys Rev Lett, 2008, 101: 107007

21 Guo J, Jin S, Wang G. et al. Superconductivity in the iron selenide $\mathrm{K}_{x} \mathrm{Fe}_{2} \mathrm{Se}_{2}(0 \leqslant x \leqslant 1.0)$. Phys Rev B, 2010, 82: 180520(R)

22 Krzton-Maziopa A, Shermadini Z, Pomjakushina E, et al. Synthesis and crystal growth of $\mathrm{Cs}_{0.8}\left(\mathrm{FeSe}_{0.98}\right)_{2}$ : a new iron-based superconductor with $T_{\mathrm{c}}=27 \mathrm{~K}$. J Phys Condens Matter, 2011, 23: 052203

23 Wang AF, Ying JJ, Yan YJ, et al. Superconductivity at $32 \mathrm{~K}$ in single-crystalline $\mathrm{Rb}_{x} \mathrm{Fe}_{2-y} \mathrm{Se}_{2}$. Phys Rev B, 2011, 83: 060512

24 Fang $\mathrm{MH}$, Wang $\mathrm{HD}$, Dong $\mathrm{CH}$, et al. Fe-based superconductivity with $T_{\mathrm{c}}=31 \mathrm{~K}$ bordering an antiferromagnetic insulator in $(\mathrm{Tl}, \mathrm{K})$ $\mathrm{Fe}_{x} \mathrm{Se}_{2}$. EPL, 2011, 94: 27009

25 Kudo K, Nishikubo Y, Nohara M. Coexistence of superconductivity and charge density wave in $\mathrm{SrPt}_{2} \mathrm{As}_{2}$. J Phys Soc Jpn, 2010, 79: 123710

26 Anand VK, Kim H, Tanatar MA, et al. Superconducting and normal-state properties of $\mathrm{APd}_{2} \mathrm{As}_{2}(\mathrm{~A}=\mathrm{Ca}, \mathrm{Sr}, \mathrm{Ba})$ single crystals. Phys Rev B, 2013, 87: 224510

27 Ying TP, Chen XL, Wang G, et al. Observation of superconductivity at 30-46 $\mathrm{K}$ in $\mathrm{A}_{x} \mathrm{Fe}_{2} \mathrm{Se}_{2}(\mathrm{~A}=\mathrm{Li}, \mathrm{Na}, \mathrm{Ba}, \mathrm{Sr}, \mathrm{Ca}, \mathrm{Yb}$, and $\mathrm{Eu})$. Sci Rep, 2012, 2: 426

28 Burrard-Lucas M, Free DG, Sedlmaier SJ, et al. Enhancement of the superconducting transition temperature of FeSe by intercalation of a molecular spacer layer. Nat Mater, 2013, 12: 15-19

29 Scheidt EW, Hathwar VR, Schmitz D, et al. Superconductivity at

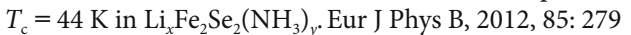

30 Tegel M, Johansson S, Weiss V, et al. Synthesis, crystal structure and spin-density-wave anomaly of the iron arsenide-fluoride SrFeAsF. EPL, 2008, 84: 67007

31 Matsuishi S, Inoue Y, Nomura T, et al. Superconductivity induced by co-doping in quaternary fluoroarsenide CaFeAsF. J Am Chem Soc, 2008, 130: 14428-14429

32 Hanna T, Muraba Y, Matsuishi S, et al. Hydrogen in layered iron arsenides: indirect electron doping to induce superconductivity. Phys Rev B, 2011, 84: 024521

33 Wu G, Xie YL, Chen H, et al. Superconductivity at $56 \mathrm{~K}$ in samarium-doped SrFeAsF. J Phys Condens Matter, 2009, 21: 142203

34 Zhu XY, Han F, Mu G, et al. $\mathrm{Sr}_{3} \mathrm{Sc}_{2} \mathrm{Fe}_{2} \mathrm{As}_{2} \mathrm{O}_{5}$ as a possible parent compound for FeAs-based superconductors. Phys Rev B, 2009, 79: 024516

35 Ogino H, Sato S, Kishio K, et al. Homologous series of iron pnictide oxide superconductors with extremely thick blocking layers. Appl Phys Lett, 2010, 97: 072506

36 Ogino H, Shimizu Y, Ushiyama K, et al. Superconductivity above 40 $\mathrm{K}$ observed in a new iron arsenide oxide $\left(\mathrm{Fe}_{2} \mathrm{As}_{2}\right)\left(\mathrm{Ca}_{4}(\mathrm{Mg}, \mathrm{Ti})_{3} \mathrm{O}_{\mathrm{y}}\right)$. Appl Phys Express, 2010, 3: 063103

37 Xie YL, Liu RH, Wu T, et al. Structure and physical properties of the new layered oxypnictides $\mathrm{Sr}_{4} \mathrm{Sc}_{2} \mathrm{O}_{6} \mathrm{M}_{2} \mathrm{As}_{2}(\mathrm{M}=\mathrm{Fe}$ and $\mathrm{Co})$. EPL, 2009, 86: 57007

38 Ogino H, Machida K, Yamamoto A, et al. A new homologous series of iron pnictide oxide superconductors $\left(\mathrm{Fe}_{2} \mathrm{As}_{2}\right)\left(\mathrm{Ca}_{n+2}(\mathrm{Al}, \mathrm{Ti})_{n} \mathrm{O}_{\mathrm{y}}\right)(n$ $=2,3,4)$. Supercond Sci Technol, 2010, 23: 115005

39 Kakiya S, Kudo K, Nishikubo Y, et al. Superconductivity at $38 \mathrm{~K}$ in iron-based compound with platinum-arsenide layers $\mathrm{Ca}_{10}\left(\mathrm{Pt}_{4} \mathrm{As}_{8}\right)$ 
$\left(\mathrm{Fe}_{1-x} \mathrm{Pt}_{x} \mathrm{As}_{2}\right)_{5}$. J Phys Soc Jpn, 2011, 80: 093704

$40 \mathrm{Ni}$, Jared MA, Chan BC, et al. High $T_{\mathrm{c}}$ electron doped $\mathrm{Ca}_{10}\left(\mathrm{Pt}_{3}\right.$ $\left.\mathrm{As}_{8}\right)\left(\mathrm{Fe}_{2} \mathrm{As} 2\right)_{5}$ and $\mathrm{Ca}_{10}\left(\mathrm{Pt}_{4} \mathrm{As}_{8}\right)\left(\mathrm{Fe}_{2} \mathrm{As}_{2}\right)_{5}$ superconductors with skutterudite intermediary layers. Proc Natl Acad Sci USA, 2011, 108: E1019-1026

41 Lu XF, Wang NZ, Zhang GH, et al. Superconductivity in $\mathrm{LiFeO}_{2}$ $\mathrm{Fe}_{2} \mathrm{Se}_{2}$ with anti-PbO-type spacer layers. Phys Rev B, 2013, 89: 020507(R)

42 Lu XF, Wang NZ, Wu H, et al. Coexistence of superconductivity and antiferromagnetism in $\left(\mathrm{Li}_{0.8} \mathrm{Fe}_{0.2}\right) \mathrm{OHFeSe}$ superconductor. Nat Mater, doi: 10.1038/nmat4155

43 Sun H, Woodruff DN, Cassidy SJ, et al. Controlling parameters for superconductivity in layered lithium iron hydroxide selenides. arXiv: 1408.4350

44 Sun YL, Jiang $\mathrm{H}$, Zhai HF, et al. $\mathrm{Ba}_{2} \mathrm{Ti}_{2} \mathrm{Fe}_{2} \mathrm{As}_{4} \mathrm{O}$ : a new superconductor containing $\mathrm{Fe}_{2} \mathrm{As}_{2}$ layers and $\mathrm{Ti}_{2} \mathrm{O}$ sheets. J Am Chem Soc, 2012, 134: 12893-12896

45 Chen XH, Dai PC, Feng DL, et al. Iron-based high transition temperature superconductors. Natl Sci Rev, 2014, 1: 371-395

46 Johnson PD, Xu GY, Yin WG (eds.). Iron-Based Superconductivity (Springer Series in Materials Science), Berlin: Springer, Vol. 211, 2014

47 Lee $\mathrm{CH}$, Kihou K, Iyo A, et al. Relationship between crystal structure and superconductivity in iron-based superconductors. Solid State Commun, 2012, 152: 644-648

48 Mizuguchi Y, Hara Y, Deguchi K, et al. Anion height dependence of $T_{\mathrm{c}}$ for the Fe-based superconductor. Supercond Sci Technol, 2010, 23: 054013

49 McQueen TM, Huang, Q, Ksenofontov V, et al. Extreme sensitivity of superconductivity to stoichiometry in $\mathrm{Fe}_{1+\delta} \mathrm{Se}$. Phys Rev B, 2009, 79: 014522

50 Iimura S, Satuishi S, Sato H, et al. Two-dome structure in electrondoped iron arsenide superconductors. Nat Commun, 2012, 3: 943

51 Drew AJ, Niedermayer C, Baker PJ, et al. Coexistence of static magnetism and superconductivity in $\mathrm{SmFeAsO}_{1-x} \mathrm{~F}_{x}$ as revealed by muon spin rotation. Nat Mater, 2009, 8: 310-314

52 Zhao J, Huang Q, Cruz C, et al. Structural and magnetic phase diagram of $\mathrm{CeFeAsO}_{1-x} \mathrm{~F}_{x}$ and its relation to high-temperature superconductivity. Nat Mater, 2008, 7: 953-959

53 Chen $\mathrm{H}$, Ren Y, Bao W, et al. Coexistence of the spin-density wave and superconductivity in $\mathrm{Ba}_{1-x} \mathrm{~K}_{x} \mathrm{Fe}_{2} \mathrm{As}_{2}$. EPL, 2009, 85: 17006

54 Nandi S, Kim MG, Kreyssig A, et al. Anomalous suppression of the orthorhombic distortion in superconducting $\mathrm{Ba}\left(\mathrm{Fe}_{1-x} \mathrm{Co}_{x}\right)_{2} \mathrm{As}_{2}$. Phys Rev Lett, 2010, 104: 057006

55 Kasahara S, Shi HJ, Hashimoto K, et al. Electronic nematicity above the structural and superconducting transition in $\mathrm{BaFe}_{2}\left(\mathrm{As}_{1-x} \mathrm{P}_{x}\right)_{2}$. Nature, 2012, 486: 382-385

56 Wang AF, Ying JJ, Wang AF, et al. A crossover in the phase diagram of $\mathrm{NaFe}_{1-x} \mathrm{Co}_{x}$ As determined by electronic transport measurements. New J Phys, 2013, 15: 043048

57 Ye ZR, Zhang Y, Chen F, et al. Extraordinary doping effects on quasiparticle scattering and bandwidth in iron-based superconductors. Phys Rev X, 2014, 4: 031041

58 Mizuguchi Y, Takano Y. Review of Fe chalcogenides as the simplest Fe-based superconductor. J Phys Soc Jpn, 2010, 79: 102001

59 Dong J, Zhang HJ, Xu G, et al. Competing orders and spin-density-wave instability in $\mathrm{La}\left(\mathrm{O}_{1-x} \mathrm{~F}_{x}\right) \mathrm{FeAs}$. EPL, 2008, 83: 27006

60 Lumsden MD, Christianson AD. Magnetism in Fe-based superconductors. J Phys Condens Matt, 2010, 22: 203203

61 De la Cruz C, Huang Q, Lynn JW et al. Magnetic order close to superconductivity in the iron-based layered $\mathrm{LaO}_{1-x} \mathrm{~F}_{x} \mathrm{FeAs}$ systems. Nature, 2008, 453: 899-902

62 Lee PA, Nagaosa N, Wen XG. Doping a Mott insulator: physics of high-temperature superconductivity. Rev Mod Phys, 2006, 78: $17-85$
63 Richard P, Sato T, Nakayama K, et al. Fe-based superconductors: an angle-resolved photoemission spectroscopy perspective. Rep Prog Phys, 2011, 74: 124512

64 Mazin II, Singh DJ, Johannes MD, et al. Unconventional superconductivity with a sign reversal in the order parameter of $\mathrm{LaFeAsO}_{1-x}$ $\mathrm{F}_{x}$. Phys Rev Lett, 2008, 101: 057003

65 Kuroki K, Onari S, Arita R, et al. Unconventional pairing originating from the disconnected Fermi surfaces of superconducting La$\mathrm{FeAsO}_{1-x} \mathrm{~F}_{x}$. Phys Rev Lett, 2008, 101: 087004

66 Wang F, Lee DH. Functional renormalization-group study of the pairing symmetry and pairing mechanism of the FeAs-based high-temperature superconductor. Phys Rev Lett, 2009, 102: 047005

67 Yao ZJ, Li JX, Wang ZD. Spin fluctuations, interband coupling and unconventional pairing in iron-based superconductors. New J Phys, 2009, 11: 025009

68 Dai PC, Hu J, Dagotto E. Magnetism and its microscopic origin in iron-based high-temperature superconductors. Nat Phys, 2012, 8: 709-718

69 Zhao J, Ratcliff W, II, et al. Spin and lattice structures of single-crystalline $\mathrm{SrFe}_{2} \mathrm{As}_{2}$. Phys Rev B, 2008, 78: 140504(R)

70 Li SL, de la Cruz C, Huang Q, et al. First-order magnetic and structural phase transitions in $\mathrm{Fe}_{1+y} \mathrm{Se}_{x} \mathrm{Te}_{1-x}$. Phys Rev B, 2009, 79: 054503

71 Bao W, Qiu Y, Huang Q, et al. Tunable $(\delta \pi, \delta \pi)$-type antiferromagnetic order in $\alpha-\mathrm{Fe}(\mathrm{Te}, \mathrm{Se})$ superconductors. Phys Rev Lett, 2009, 102: 247001

72 Bao W, Huang Q, Chen GF, et al. A novel large moment antiferromagnetic order in $\mathrm{K}_{0.8} \mathrm{Fe}_{1.6} \mathrm{Se}_{2}$ superconductor. Chin Phys Lett, 2011 , 28: 086104

73 Liu RH, Wu G, Wu T, et al. Anomalous transport properties and phase diagram of the FeAs-based $\mathrm{SmFeAsO}_{1-x} \mathrm{~F}_{x}$ superconductors. Phys Rev Lett, 2008, 101: 087001

74 Yan YJ, Wang AF, Luo XG, et al. Power-law temperature dependent hall angle in the normal state and its correlation with superconductivity in iron-pnictides. arXiv: 1301.1734

75 Fang C, Yao H, Tsai WF, et al. Theory of electron nematic order in LaFeAsO. Phys Rev B, 2008, 77: 224509

76 Yildirim T. Strong coupling of the Fe-spin state and the As-As hybridization in iron-pnictide superconductors from first-principle calculations. Phys Rev Lett, 2009, 102: 037003

77 Xu C, Muller M, Sachdev S. Ising and spin orders in the iron-based superconductors. Phys Rev B, 2008, 78: 020501

78 Johannes M, Mazin II. Microscopic origin of magnetism and magnetic interactions in ferropnictides. Phys Rev B, 2009, 79: 220510

79 Wang QY, Li Z, Zhang WH, et al. Interface-induced high-temperature superconductivity in single unit-cell FeSe films on $\mathrm{SrTiO}_{3}$. Chin Phys Lett, 2012, 29: 037402

80 Liu DF, Zhang WH, Mou WX, et al. Electronic origin of high-temperature superconductivity in single-layer FeSe superconductor. Nat Commun, 2012, 3: 931

81 He SL, He JF, Zhang WH, et al. Phase diagram and electronic indication of high-temperature superconductivity at $65 \mathrm{~K}$ in single-layer FeSe films. Nat Mater, 2013, 12: 605-610

82 Tan SY, Zhang Y, Xia M, et al. Interface-induced superconductivity and strain-dependent spin density waves in $\mathrm{FeSe} / \mathrm{SrTiO}_{3}$ thin films. Nat Mater, 2013, 12: 634-640

83 Ge JF, Liu ZL, Liu CH, et al. Superconductivity above $100 \mathrm{~K}$ in single-layer FeSe films on doped $\mathrm{SrTiO}_{3}$. Nat Mater, doi:10.1038/ nmat4153

84 Luetkens $\mathrm{H}$, Klauss $\mathrm{HH}$, Kraken $\mathrm{M}$, et al. The electronic phase diagram of the $\mathrm{LaO}_{1-x} \mathrm{~F}_{x} \mathrm{FeAs}$ superconductor. Nat Mater, 2009, 8: 305-309

85 Rotundu CR, Keane DT, Freelon B, et al. Phase diagram of the Pr$\mathrm{FeAsO}_{1-x} \mathrm{~F}_{x}$ superconductor. Phys Rev B, 2009, 80: 144517

86 Malavasi L, Artioli GA, Ritter C, et al. Phase diagram of NdFeAs $\mathrm{O}_{1-x} \mathrm{~F}_{x}$ : essential role of chemical. J Am Chem Soc, 2010, 132: 2417- 
2420

87 Fukazawa H, Yamazaki T, Kondo K, et al. ${ }^{75}$ As NMR study of holedoped superconductor $\mathrm{Ba}_{1-x} \mathrm{~K}_{x} \mathrm{Fe}_{2} \mathrm{As}_{2}\left(T_{\mathrm{c}}\right.$ similar or equal to $\left.38 \mathrm{~K}\right)$. J Phys Soc Jpn, 2009, 78: 033704

88 Park JT, Inosov DS, Niedermayer C, et al. Electronic phase separation in the slightly underdoped iron pnictide superconductor $\mathrm{Ba}_{1-x}$ $\mathrm{K}_{x} \mathrm{Fe}_{2} \mathrm{As}_{2}$. Phys Rev Lett, 2009, 102: 117006

89 Rotter M, Tegel M, Schellenberg I, et al. Competition of magnetism and superconductivity in underdoped $\left(\mathrm{Ba}_{1-x} \mathrm{~K}_{x}\right) \mathrm{Fe}_{2} \mathrm{As}_{2}$. New J Phys, 2009, 11: 025014

90 Li Z, Zhou R, Liu Y, et al. Microscopic coexistence of antiferromagnetic order and superconductivity in $\mathrm{Ba}_{0.77} \mathrm{~K}_{0.23} \mathrm{Fe}_{2} \mathrm{As}_{2}$. Phys Rev $\mathrm{B}$, 2012, 86: 180501(R)

91 Laplace Y, Bobroff J, Rullier-Albenque F, et al. Atomic coexistence of superconductivity and incommensurate magnetic order in the pnictide $\mathrm{Ba}\left(\mathrm{Fe}_{1-x} \mathrm{Co}_{x}\right)_{2} \mathrm{As}_{2}$. Phys Rev B, 2009, 80: 140501

92 Bernhard C, Drew AJ, Schulz L, et al. Muon spin rotation study of magnetism and superconductivity in $\mathrm{BaFe}_{2-x} \mathrm{Co}_{x} \mathrm{As}_{2}$ and $\mathrm{Pr}_{1-x} \mathrm{Sr}_{x-}$ FeAsO. New J Phys, 2009, 11: 055050

93 Ma L, Ji GF, Dai J, et al. Microscopic coexistence of superconductivity and antiferromagnetism in underdoped $\mathrm{Ba}\left(\mathrm{Fe}_{1-x} \mathrm{Ru}_{x}\right)_{2} \mathrm{As}_{2}$. Phys Rev Lett, 2012, 109: 197002

94 Pratt DK, Tian W, Kreyssig A, et al. Coexistence of competing antiferromagnetic and superconducting phases in the underdoped $\mathrm{Ba}\left(\mathrm{Fe}_{0.953} \mathrm{Co}_{0.047}\right)_{2} \mathrm{As}_{2}$ compound using X-ray and neutron scattering techniques. Phys Rev Lett, 2009, 103: 087001

95 Christianson AD, Lumsden MD, Nagler SE, et al. Static and dynamic magnetism in underdoped superconductor $\mathrm{BaFe}_{1.92} \mathrm{Co}_{0.08} \mathrm{As}_{2}$. Phys Rev Lett, 2009, 103: 087002

96 Jiang S, Xing H, Xuan GF, et al. Superconductivity up to $30 \mathrm{~K}$ in the vicinity of the quantum critical point in $\mathrm{BaFe}_{2}\left(\mathrm{As}_{1-x} \mathrm{P}_{x}\right)_{2}$. J Phys Condens Matter, 2009, 21: 382203

97 Kasahara S, Shibauchi T, Hashimoto K, et al. Evolution from non-Fermi- to Fermi-liquid transport via isovalent doping in $\mathrm{BaFe}_{2}\left(\mathrm{As}_{1-x} \mathrm{P}_{x}\right)_{2}$ superconductors. Phys Rev B, 2010, 81: 184519

98 Lohneysen HV, Rosch A, Vojta M, et al. Fermi-liquid instabilities at magnetic quantum phase transitions. Rev Mod Phys, 2007, 79: 1015

99 Fisher IR, Degiorgi L, Shen ZX, et al. In-plane electronic anisotropy of underdoped ' 122 ' Fe-arsenide superconductors revealed by measurements of detwinned single crystals. Rep Prog Phys, 2011, 74: 124506

100 Chu JH, Analytis JG, De Greve K, et al. In-plane resistivity anisotropy in an underdoped iron arsenide superconductor. Science, 2010, 329: 824-826

101 Ying JJ, Wang XF, Wu T, et al. Measurements of the anisotropic inplane resistivity of underdoped FeAs-based pnictide superconductors. Phys Rev Lett, 2011, 107: 067001

102 Ishida S, Nakajima M, Liang T, et al. Effect of doping on the magnetostructural ordered phase of iron arsenides: a comparative study of the resistivity anisotropy in doped $\mathrm{BaFe}_{2} \mathrm{As}_{2}$ with doping into three different sites. J Am Chem Soc, 2013, 135: 3158-3163

103 Blomberg EC, Tanatar MA, Fernandes RM, et al. Sign-reversal of the in-plane resistivity anisotropy in hole-doped iron pnictides. Nat Commun, 2013, 4: 1914

104 Ma JQ, Luo XG, Cheng P, et al. Evolution of anisotropic in-plane resistivity with doping level in $\mathrm{Ca}_{1-x} \mathrm{Na}_{x} \mathrm{Fe}_{2} \mathrm{As}_{2}$ single crystals. Phys Rev B, 2014, 89: 174512

105 Ishida S, Nakajima M, Liang T, et al Anisotropy of the in-plane resistivity of underdoped $\mathrm{Ba}\left(\mathrm{Fe}_{1-x} \mathrm{Co}_{x}\right)_{2} \mathrm{As}_{2}$ superconductors induced by impurity scattering in the antiferromagnetic orthorhombic phase. Phys Rev Lett, 2013, 110: 207001

106 Allan MP, Chuang TM, Masseeet F, et al. Anisotropic impurity states, quasiparticle scattering and nematic transport inunderdoped $\mathrm{Ca}\left(\mathrm{Fe}_{1-x} \mathrm{Co}_{x}\right) \mathrm{As}_{2}$. Nat Phys, 2013, 9: 220-224

107 Zhang, LJ, Singh DJ, Du MH, et al. Density functional study of FeS,
FeSe, and FeTe: electronic structure, magnetism, phonons, and superconductivity. Phys Rev B, 2008, 78: 134514

108 Xia Y, Qian D, Wray L, et al. Fermi surface topology and low-lying quasiparticle dynamics of parent $\mathrm{Fe}_{1-x} \mathrm{Te} / \mathrm{Se}$ superconductor. Phys Rev Lett, 2009, 103: 037002

109 Liu TJ, Ke X, Qian B, et al. Charge-carrier localization induced by excess Fe in the superconductor $\mathrm{Fe}_{1+y} \mathrm{Te}_{1-x} \mathrm{Se}_{x}$. Phys Rev B, 2009, 80: 174509

110 Paulose PL, Yadav CS, Subhedar KM. Magnetic phase diagram of $\mathrm{Fe}_{1,1} \mathrm{Te}_{1-x} \mathrm{Se}_{x}$ : a comparative study with the stoichiometric superconducting $\mathrm{FeTe}_{1-x} \mathrm{Se}_{x}$ system. EPL, 2010, 90: 27011

111 Margadonna S, Takabayashi Y, McDonald MT, et al. Crystal structure of the new $\mathrm{FeSe}_{1-x}$ superconductor. Chem Commun, 2008, 5607-5609

112 Stewart GR. Superconductivity in iron compounds. Rev Mod Phys, 2011, 83: 1589

113 Takahashi H, Okada H, Kamihara Y, et al. Pressure effect of superconducting oxypnictide $\mathrm{LaFeAO}_{1-x} \mathrm{~F}_{x}$ and related materials. J Phys Conf Ser, 2010, 215: 012037

114 Colombier E, Torikachvili MS, Ni N, et al. Electrical transport measurements under pressure for $\mathrm{BaFe}_{2} \mathrm{As}_{2}$ compounds doped with $\mathrm{Co}$, Cr, or Sn. Supercond Sci Technol, 2010, 23: 054003

115 Zhang SJ, Wang XC, Liu QQ, et al. Superconductivity at $31 \mathrm{~K}$ in the "111"-type iron arsenide superconductor $\mathrm{Na}_{1-x} \mathrm{FeAs}$ induced by pressure. EPL, 2009, 88: 47008

116 Wang AF, Xiang ZJ, Ying JJ, et al. Pressure effects on the superconducting properties of single-crystalline Co doped NaFeAs. New J Phys, 2012, 14: 113043

117 Alireza PL, Chris Ko YT, Gillett J, et al. Superconductivity up to $29 \mathrm{~K}$ in $\mathrm{SrFe}_{2} \mathrm{As}_{2}$ and $\mathrm{BaFe}_{2} \mathrm{As}_{2}$ at high pressures. J Phys Condens Matter, 2009, 21: 012208

118 Miclea CF, Nicklas M, Jeevan HS, et al. Evidence for a reentrant superconducting state in $\mathrm{EuFe}_{2} \mathrm{As}_{2}$ under pressure. Phys Rev B, 2009, 79: 212509

119 Ahilan K, Ning FL, Imai T, et al. Electronic phase diagram of the iron-based high- $T_{\mathrm{c}}$ superconductor $\mathrm{Ba}\left(\mathrm{Fe}_{1-x} \mathrm{Co}_{x}\right)_{2} \mathrm{As}_{2}$ under hydrostatic pressure $(0 \leqslant x \leqslant 0.099)$. Phys Rev B, 2009, 79: 214520

120 Zhang M, Ying JJ, Yan YJ, et al. Phase diagram as a function of doping level and pressure in $\mathrm{Eu}_{1-x} \mathrm{La}_{x} \mathrm{Fe}_{2} \mathrm{As}_{2}$ system. Phys Rev B, 2012, 85: 092503

121 Lu XF, Wang NZ, Chen XH, et al. Superconductivity and phase diagram in $\left(\mathrm{Li}_{0.8} \mathrm{Fe}_{0.2}\right) \mathrm{OHFeSe} \mathrm{H}_{1-x} \mathrm{~S}_{x}$. Phys Rev B, 2014, 90: 214520

122 Ying JJ, Wang XF, Luo XG, et al. Pressure effect on superconductivity of $\mathrm{A}_{x} \mathrm{Fe}_{2} \mathrm{Se}_{2}$ (A = K and Cs). New J Phys, 2011, 13: 033008

123 Sun LL, Chen XJ, Guo J, et al. Re-emerging superconductivity at 48 kelvin in iron chalcogenides. Nature, 2012, 483: 67-69

124 Guo J, Chen XJ, Dai JH, et al. Pressure-driven quantum criticality in iron-selenide superconductors. Phys Rev Lett, 2012, 108: 197001

125 Lai KT, Takemori A, Miyasaka S, et al. Suppression of superconductivity around $x=0.5-0.7$ in $\mathrm{LaFe}_{1-x} \mathrm{As}_{x} \mathrm{O}_{0.95} \mathrm{~F}_{0.05}$. JPS Conf Proc, 2014, 1: 012104

126 Okabe H, Takeshita N, Horigane K, Muranaka T, Akimitsu J. Pressure-induced high- $T_{\mathrm{c}}$ superconducting phase in FeSe: correlation between anion height and $T_{c}$. Phys Rev B, 2010, 81: 205119

127 Lee $\mathrm{CH}$, Iyo A, Eisaki $\mathrm{H}$, et al. Effect of structural parameters on superconductivity in fluorine-free $\mathrm{LnFeAsO}_{1-y}(\mathrm{Ln}=\mathrm{La}, \mathrm{Nd})$. J Phys Soc Jpn, 2008, 77: 083704

128 Kumai R, Takeshita N, Ito T, et al. Pressure-induced modification of crystal structure in $\mathrm{NdFeAsO}_{1-y}(1-y=0.85)$, accompanied by remarkable suppression of $T_{c}$. J Phys Soc Jpn, 2009, 78: 013705

Acknowledgement This work was supported by the National Natural Science Foundation of China (11190021, U1330105, 11174266), the "Strategic Priority Research Program (B)" of the Chinese Academy of Sciences (XDB04040100), and the National Basic Research Program of 
China (2011CBA00101).

Author contributions Luo XG and Chen XH wrote the paper; Chen $\mathrm{XH}$ provided the overall concept and revised the manuscript. Both of the authors participated in the discussion.
Conflict of interest The authors declare that they have no conflict of interest.

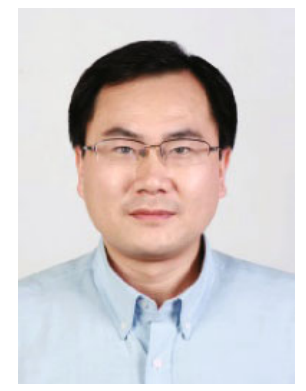

Xigang Luo is an associate Professor in Physics at the University of Science and Technology of China (USTC). He received his $\mathrm{PhD}$ degree from USTC in 2005. His research is focused on the study of the physics of novel functional materials, such as unconventional superconductors, thermoelectric oxides.

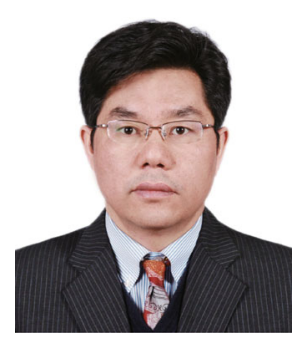

Xianhui Chen obtained his PhD in physics from the University of Science and Technology of China (USTC) in 1992. In the same year, he began his research career in USTC and now holds the position of Professor in Physics. His research is focused on the exploration and study of the physics of novel functional materials exhibiting superconductivity, novel magnetism, thermoelectricity, etc.

中文摘要 自铁基化合物中发现高温超导电性以来, 人们已经合成了众多具有不同间隔层的体系. 同时科学家为揭示铁基超导体高温 超导电性的特征和起源, 在实验和理论研究方面都投入了大量的精力. 但是直到今天其高温超导电性的起源仍然没有得以解决, 特别 是对正常态性质背后的物理还存在很多争论. 本文总结了铁基超导体系的晶体结构以及相图, 并提供了一些指向具有更高转变温度 $T_{\mathrm{c}}$ 的新超导体的提示和高温超导机理的线索. 